\title{
Diversidad cultural en la escuela pública colombiana 1960-2010
}

Cultural diversity in the colombian public school 1960-2010 Diversidade cultural na escola pública colombiana 1960-2010 Marco Tulio Cárdenas Forero Camila Urueta Gutiérrez 


\section{Marco Tulio Cárdenas Forero ${ }^{1}$ Camila Urueta Gutiérrez ${ }^{2}$}

1 Secretaria de Educación Distrital de Bogotá, Magister en Desarrollo educativo y Social CINDE - Universidad Pedagógica Nacional.

2 Ministerio de Educación Nacional, Magister en Desarrollo educativo y Social CINDE Universidad Pedagógica Nacional.

\section{Resumen}

El presente artículo contiene los resultados de un proyecto de investigación realizado bajo la perspectiva arqueológicogenealógica; se busca realizar un aporte a las políticas del conocimiento en educación y pedagogía, proponiendo un nuevo debate sobre el tema de la diversidad cultural. Básicamente se trata del producto de una excavación en el archivo histórico del país, llevada a cabo para indagar la manera en que la diversidad cultural se ha instaurado en la escuela pública colombiana entre 1960 y 2010, y ver cómo dicho enunciado ha hecho de ella un saber escolar.

\section{Palabras clave: Diversidad cultural,} arqueología-genealogía, saberes escolares, fuerzas, subjetividades.

\section{Summary}

This article shows the results of a research project done under an archaeological-genealogical perspective study promotes a new debate around cultural diversity - hold in different discussions in a social level- with the aim to contribute to the knowledge of policies in education and pedagogy. Thus, this is the result of an excavation of the Colombian historical records carried out in order to investigate the way cultural diversity has been established in the Colombian public school between 1960 and 2010, and understand how this statement has made of it, a school learning

\section{Keywords: Cultural diversity,} archaeology-genealogy, school knowledge, forces, subjectivities.

\section{Resumo}

Este artigo apresenta os resultados de um projecto de investigação realizado sob a perspectiva arqueológica e pesquisa genealógica que contribui para as políticas do conhecimento em educação e pedagogia um novo debate sobre o tema da diversidade cultural presente as discussões de ordem social. Ela própria é o produto de uma escavação no arquivo histórico do país, que foi realizado com o objetivo de investigar a forma em que ele estabeleceu a diversidade cultural na escola pública da Colômbia entre os anos 1960 e 2010, e veja como a declaração foi feita, um conhecimento escolar.

Palavras chave: Diversidade cultural, arqueologia e genealogia, o conhecimento escolar, as forças, as subjetividades. 


\section{Introducción}

os planteamientos expuestos en el presente artículo están de_sarrollados dentro del enfoque posestructuralista, particularmente bajo la propuesta teórica de la obra de Michel Foucault. Se trata de un rastreo arqueológico-genealógico realizado sobre archivo histórico de Colombia, hecho con el objeto de mostrar algunos enunciados que se han posicionado como verdades en torno a la diversidad cultural en la escuela, y determinar las relaciones que existen entre las políticas del conocimiento en educación y pedagogía, los saberes escolares y la diversidad cultural en la escuela colombiana.

La inmersión en este enfoque no busca causas y efectos de fenómenos sociales, sino fuerzas y tensiones entre objetos, sujetos, prácticas e instituciones, enmarcadas en luchas que han objetivado algunos discursos como el de la diversidad cultural en la escuela pública colombiana.

El ejercicio investigativo implicó problematizar no sólo las verdades constituidas, sino también la postura y el ejercicio propios del investigador, para intentar ver de otra forma los problemas presentes en la educación del país, tomar distancia de toda interpretación para entender el modo de ser de la escuela de hoy.

\section{Diversidad cultural en la escuela de hoy: una apuesta al reconocimiento de la diferencia}

En los inicios de la segunda mitad del siglo XX, con la explosión de pactos internacionales que obligaban a las naciones firmantes a reconocer derechos individuales y colectivos, toman cada vez más importancia ideas como la no exclusión por razones de raza. La nueva mirada al alumno como un sujeto al que se le deben atender sus necesidades y características individuales, la emergencia de nuevos modelos educativos que buscaban llegar a la población marginada lejos de las grandes urbes, y sobre todo, los movimientos indígenas de reivindicación de derechos, particularmente en busca de la recuperación de sus territorios colectivos -cuestiones que serán expuestas en su momento-, se convirtieron en algunos de los enunciados fuerza que permitieron la emergencia de la diversidad cultural en la escuela.
Así, desde los años 70 comienzan a ser visibles prácticas y mandatos que ponían en juego la diversidad en la institución escolar. Primero, bajo lo que se denomina etnoeducación, el enunciado de diversidad entró con fuerza para reivindicar derechos como el de la atención educativa especial para pueblos indígenas, o el de la autonomía de las etnias para definir sus intereses y necesidades culturales; segundo, dos décadas más adelante, el enunciado giró hacia la necesidad de hablar de interculturalidad como de la relación entre culturas, más allá del reconocimiento individual a cada una de ellas, idea que en la actualidad se intenta objetivar.

El Ministerio de Educación Nacional, con el Decreto 088 de 1976, comenzó a hablar de programas para comunidades indígenas que buscaran la conservación y divulgación de su cultura. Dos años después, reglamentando la educación para pueblos indígenas, por medio del Decreto 1142, se empezó a hablar de una educación que estuviera relacionada con su medio ambiente, con sus procesos productivos y, por supuesto, con la vida social y cultural de cada una de ellos. De esta manera, los programas curriculares debían asegurar "el respeto y el fomento de su patrimonio económico, natural, cultural y social, sus valores artísticos, sus medios de expresión y sus creencias religiosas" (Ministerio de Educación Nacional, 1978).

Al tiempo, la educación afrocolombiana también surgía para combatir la discriminación racial, defender los derechos culturales y la equidad entre pueblos. Así, en 1977 se realizaría en la ciudad de Cali el Primer Congreso de Cultura Negra de las Américas, en donde se puso sobre la mesa la importancia de investigar sobre los elementos culturales de procedencia africana e incluirlos en los currículos escolares. Las conclusiones del evento, todas ellas sobre enseñanza, cultura e identidad, se convirtieron en reflexiones que guiaron las prácticas educativas afrocolombianas surgidas años más tarde:

"[...] pues aún cuando el Estado colombiano estableció políticas y prácticas de reconocimiento de los pueblos indígenas, a los afrocolombianos no se les reconoce como grupo étnico sino hasta la Constitución de 1991, y con mayor claridad en la Ley 70 de 1993.

Estas normas recogen la síntesis de los planteamientos sobre

educación que se dieron en el Primer Congreso de Cultura Negra de las Américas, de 1977 (UNESCO, 2005, p. 292).

Para entonces la UNESCO publicaba la declaración mundial sobre la raza y los prejuicios raciales, centrada en la afirmación de que: "todos los seres humanos pertenecen a la misma especie y tienen el mismo origen", por lo tanto "nacen iguales en 
dignidad y derechos" (UNESCO, 1978). Con esto como base, la Constitución Política de Colombia de 1991, acogería como principio del estado colombiano, y hasta la actualidad que:

"Todas las personas nacen libres e iguales ante la ley, recibirán

la misma protección y trato de las autoridades y gozarán de los mismos derechos, libertades y oportunidades sin ninguna discriminación por razones de sexo, raza, origen nacional o familiar, lengua, religión, opinión política o filosófica” (Presidencia de la República, 1991).

Para 1982, la Revista Educación de Territorios Nacionales, publicaría los "Lineamientos generales de la educación indígena definidos por el Ministerio de Educación Nacional", allí se comienza a reconocer la cultura propia como: "el marco referencial de todas las acciones de la etnoeducación, en cuanto orienta la fijación de objetivos, define procesos y metodologías, precisa estrategias y determina los manejos administrativos" (Alfonso, 1982).

En ese sentido, la comunidad comenzaría a jugar un papel primordial en los procesos educativos para los grupos étnicos, ya que la definición de los diversos componentes, así como la ejecución de los proyectos de etnoeducación, estarían bajo responsabilidad de cada comunidad y sus resultados como tal debían ser el esfuerzo y la acción conjunta de la población al interior de éstas.

Para 1990, UNESCO, UNICEF, PNUD, FNUAP y el Banco Mundial, insistían en que las necesidades básicas de aprendizaje varían con cada cultura y cambian con el tiempo; en tal sentido, la educación primaria debía tener en cuenta la cultura, es decir que dichas necesidades debían ser satisfechas para lograr el respeto y enriquecimiento de la herencia cultural, lingüística y espiritual común, y transmitir los valores culturales y morales. Así mismo, afirmaron que la educación, vista como acceso a los conocimientos, podría contribuir al progreso y los cambios culturales, siempre y cuando se generaran programas que lograran suprimir barreras sociales y culturales, y buscaran preservar la identidad (Naciones Unidas, 1990).

Así, en 1997, el Ministerio de Educación Nacional, mediante el Acta 115, creó el grupo de Etnoeducación, cuya tarea principal sería la de impulsar programas educativos especiales dirigidos a los grupos étnicos. Con esto, un año más tarde la Corte Suprema de Justicia podría afirmar que el reconocimiento a la diversidad se da por el interés de construir:

"[...] una democracia cada vez más inclusiva y participativa y de ser consecuentes, de otro lado, en la concepción según la cual la justicia constituye un ideal incompleto si no atiende a las reivin- dicaciones de reconocimiento de los individuos y comunidades" (Sentencia 510, 1998).

Lo anterior se fortalece cuando en el año 2000, el Pronunciamiento Latinoamericano, dando mayor fuerza al enunciado de la diversidad cultural en la escuela pública, afirmó que las "naciones son pueblos de pueblos, gestados a través de procesos de mestizaje biológico y cultural, por lo que la apertura a la pluralidad de razas, etnias y culturas es esencial a nuestra identidad y valor, a ser reafirmado por la educación" (Pronunciamiento Latinoamericano, 2000, pp. 4-6).

Por su parte, la UNESCO afirmaría que la educación intercultural pareciera ser, por definición, la encargada de liderar procesos de valoración de la diversidad cultural en el ámbito escolar, ya que es un enfoque que se basa en las diferencias culturales como: "recurso para el fortalecimiento de la propia identidad y, al mismo tiempo, reconociendo a la otra cultura como valiosa y digna de aprecio" (UNESCO, 2002).

La emergencia de la interculturalidad en la escuela empieza a tener distintas miradas, es el caso de la Convención sobre la Diversidad de las Expresiones Culturales (2005), donde surgió una definición de interculturalidad que haría referencia "a la presencia e interacción equitativa de diversas culturas y la posibilidad de generar expresiones culturales compartidas, adquiridas por medio del diálogo y de una actitud de respeto mutuo" (UNESCO, 2005), complementando así la mirada desde las políticas culturales que entienden la interculturalidad como un proceso social y político mediante el cual se respetan, influyen y transforman de manera permanente las relaciones entre culturas.

Desde entonces, los Lineamientos de Política para la Atención Educativa a Poblaciones Vulnerables del Ministerio de Educación Nacional, plantearon que el servicio etnoeducativo se debía fundamentar, entre otros principios, en el de interculturalidad. Desde esta perspectiva, la escuela buscaría "valorizar y reforzar las identidades locales abriéndose, al mismo tiempo, al diálogo intercultural con el mundo plural y diverso, lo cual estaría a tono con el nuevo orden que surge como resultado de la mundialización” (Ministerio de Educación Nacional, 2005).

De acuerdo con lo anterior, se plantea que educar en la interculturalidad es "valorar, al mismo tiempo las diferencias culturales y utilizarlas como recurso pedagógico" (UNESCO, 2008, p. 23), basándose en la premisa de que las sociedades contemporáneas son interculturales de hecho y que sus relaciones asumen diferentes formas y diversos signos. La interculturalidad hace referencia a un ideal, a un deber ser o a un proyecto posi- 

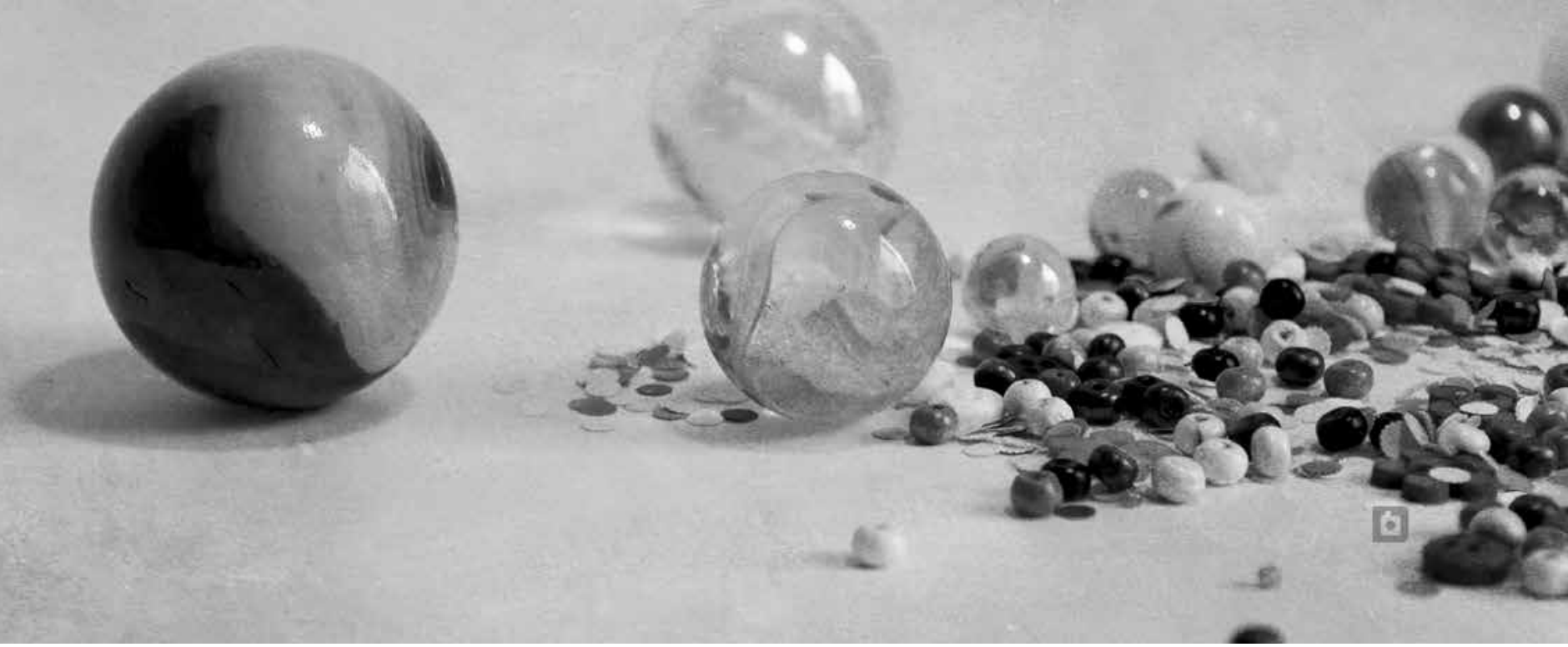

blemente utópico de mejorar las relaciones asimétricas entre las culturas para generar intercambios dialógicos. En ese sentido, habla entonces de tener en cuenta que:

"[...] no sólo es suficiente la aceptación y tolerancia de alumnos de diversas culturas en un centro escolar, sino que es necesario su reconocimiento y efectiva atención desde los diferentes componentes de la educación, el currículum y sus elementos, hasta los procesos de enseñanza aprendizaje en el aula (Escuela de Educación Diferencial, 2009, p. 115).

Es así como en la actualidad, cuando se habla de interculturalidad se expresa en términos de "una razón de vida que va más allá de la formalidad de la existencia de las comunidades indígenas y afrodescendientes" (Escuela de Educación Diferencial, 2009).

En suma, desde la década de los 70 aparece en la escuela pública colombiana el reconocimiento a la diversidad cultural; primero a partir del enunciado de la etnoeducación, que permitió pensar en la participación de la comunidad en los desarrollos curriculares, en la importancia del fortalecimiento de la cultura y en la necesidad de reconocer y respetar a los otros; segundo, desde la interculturalidad, la cual, recogiendo los fundamentos de la etnoeducación, ampliaría su mirada incluyendo el principio de reconocimiento, para fomentar relaciones e intercambios entre culturas que conviven cotidianamente en el mismo lugar.

\section{Años 60. La escuela frente a la urgencia de la unidad nacional}

Con un panorama que describe la manera en que la diversidad cultural hace parte de la escuela a través de la etnoeducación y los enfoques interculturales en la actualidad, nos preguntamos ¿Cómo era el modo de ser de la escuela antes de los 70?; ¿Concebía las diferencias culturales?; ¿qué fuerzas produjeron el modo de ser de la escuela actual?; ¿la escuela como institución objetivada para unos fines específicos, puede afrontar la incursión de la diversidad cultural?

En la década del 60, la educación impartida por la escuela primaria pública colombiana dirigida por el Estado, era considerada como "la etapa inicial del proceso educativo general y sistemático, a la que toda persona, sin discriminación alguna, tiene derecho a partir de los siete (7) años de edad" (Ministerio de Educación Nacional, 1963). Esta forma de escolarización se establecía como el lugar necesario para regular, instruir, enseñar y orientar los comportamientos, actitudes y valores de los niños y jóvenes, en últimas, para consolidar la idea de la unidad nacional que se fundamentaba en aquel tiempo como un proyecto de unificación de una sola raza, una sola lengua, una única moneda y un solo Dios. 


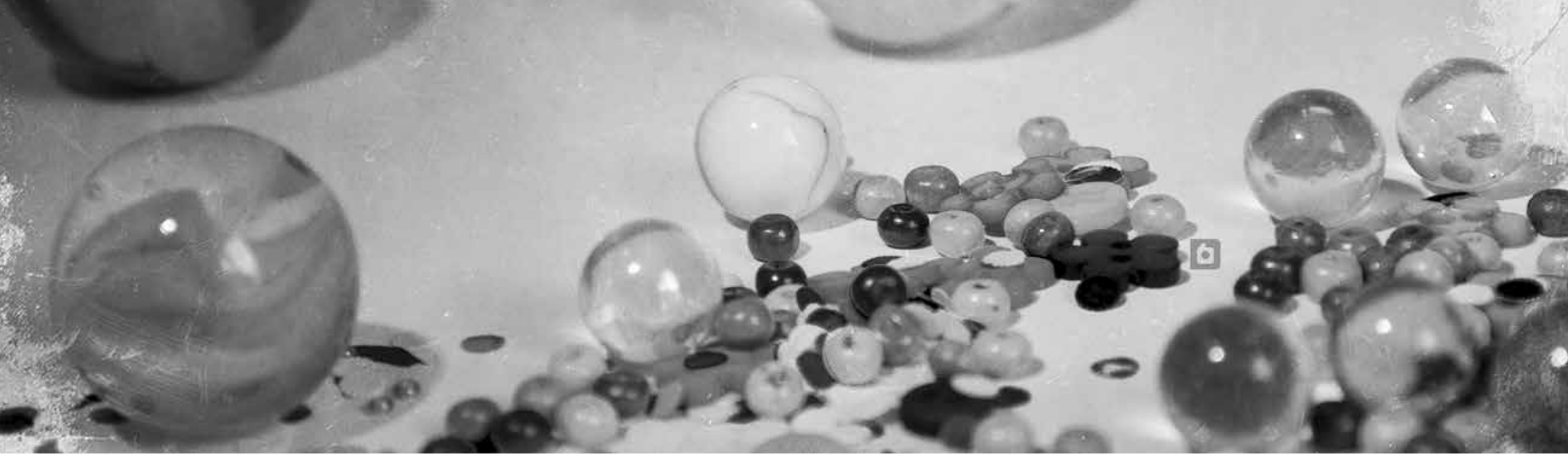

Así pues, la escuela estaba enmarcada en el pensamiento de la homogeneidad, donde lo diverso era inexistente e impensable. Esto era particularmente evidente desde tres prácticas: el texto escolar, la evaluación y la planificación educativa. Los textos escolares de finales de los años cincuenta, por ejemplo, mostraban el pensamiento homogéneo, en la medida en que consideraron que "(...) lejos de acentuarse las líneas diferenciales, los diversos grupos colombianos van hacia una relativa unificación" (Ramón, 1959, p.36). Es decir que los saberes escolares funcionaron como parte de la intención de mostrar que a pesar de las diferencias marcadas entre los pobladores del país, había que procurar la unidad.

Para los años 60 se empezaba a insinuar en dichos textos la inclusión de lo diverso, quizá todavía no de lo cultural, pero sí de las características físicas y los hábitos particulares de los sujetos de cada región, todo ello en función de un único proyecto nacional. Por ejemplo, en el texto escolar Serie El Dorado de Ciencias Sociales y Geografía, de 1969, se expresarían descripciones como,

“(...) el hombre de la montaña es nuestro campesino común y corriente. Hoy procura ilustrarse y para ello asiste a la escuela primaria, lee el periódico y sigue con atención las lecciones de Radio Sutatenza [...] el negro es muy alegre y supersticioso; su música es reflejo fiel de una raza que de continuo añora la tierra de donde lo sacaron a la fuerza [...] el isleño es educado, cortés y hospitalario y está muy pegado de la belleza de su pequeño mundo, empenachado de palmeras y sus límpidos horizontes de color cristal" (Maristas, 1969, pp. 47, 49 y 50).

En esta década, la evaluación, como práctica escolarizada, permitió determinar las diferencias entre estudiantes, desde sus formas de aprender hasta sus resultados académicos, los cuales debían ser registrados en documentos escolares. Era la evidencia que se daría a conocer a los padres y madres de familia, o acudientes. Por ello, el Decreto 45 de 1962 obligaba a todos los establecimientos educativos a adoptar "libretas escolares individuales", donde se consignarían las calificaciones y las observaciones sobre asistencia, comportamiento y esfuerzo. El entregar informes individuales se consolidaba como un mecanismo que afirmaba la unificación de criterios -planes de estudio- con lo que se pretendía para garantizar la unidad nacional, sin embargo, con ello se insinuaba la necesidad de ver a los alumnos de un modo particular, diversos.

Finalmente, el tercer elemento que caracterizó a la escuela pública de inicios de la segunda mitad del siglo XX, fue la planificación. Para entonces, la Misión Pedagógica Alemana y el Proyecto Multinacional de Tecnología Educativa, empezaron el programa de planificación de la enseñanza en la escuela primaria, el cual tenía por objeto disminuir "la desigualdad entre la zona rural y urbana en cuanto a la diferenciación de planes, contenidos básicos y duración de la escolaridad obligatoria" (Ferro, 1982, p. 3). La planificación escolar que buscaba igualmente la unificación, también propendía por una enseñanza distinta, caracterizada por el entorno donde la escuela operaba.

Con esta base, la evaluación, que estaba determinada para comprobar el logro de objetivos académicos, y la planificación escolar, que venía de escenarios distintos a la educación (planificación empresarial, tecnificación de los procesos industriales, entre otros, con una diversidad de conocimientos y saberes), impulsaron en parte el acontecimiento de la diversidad cultural dentro del escenario escolar.

Si bien las prácticas educativas "no cambiaron la estructura de la escuela pública sí transformaron procesos que se realizaban dentro de la enseñanza” (Martínez Boom, 1994, p. 56), fue en ellas, con la implementación de técnicas como la Curricularización, donde se caería en cuenta de la existencia de la diferencia, no solo de razas, sino de orden cultural y sicológico. Mostrando esto, Alberto Martínez Boom enuncia que:

"No sobra aclarar que el proceso de Curricularización de la educación, es decir, el proceso mediante el cual el campo del currículo toma el control de la dirección de la educación y la enseñanza, es un proceso en el que se reconocen matices, ritmos, niveles diferentes de desarrollo, apropiación y adecuación de las prácticas educativas" (Martínez Boom, 1994, p. 66). 
De allí que la escuela pública de los 60 y los movimientos que en ella se gestaban, fuera cambiando hacia el reconocimiento de lo diverso culturalmente hablando, desde sus prácticas -Curricularización y Tecnologización- y que como tal se instauraría desde este enunciado con un principio que la define hoy: la atención de todos los niños teniendo en cuenta sus diferencias.

En suma, en la década de los años 60 la institución escolar hacía de sus prácticas elementos que permitían reafirmar la unidad nacional, la homogenización del país. Antes de los 70 para la escuela era un problema reconocer la diferencia regional y, por supuesto, no se planteaba entonces la posibilidad de una diversidad en términos culturales. A pesar de que se reconocían las disparidades geográficas y sociales de los colombianos, estas diferencias se mostraban con el fin de fortalecer la nación, dar una identidad particular pero dentro de un todo homogéneo.

\section{Del triétnico al sujeto de derechos}

Pasada la década de los años 60, en la que los fines de la escuela se determinaban por la búsqueda del fortalecimiento de la unidad nacional (un solo dios, una sola raza), en la década del 70 comenzaron a verse pequeños cambios en la escuela que insinuaban su nuevo modo de ser, definido por el reconocimiento a la diversidad, en contravía de lo que se concebía hasta entonces. Esos movimientos fueron impulsados por diversas fuerzas provenientes de distintos campos, líneas de poder que confluyeron para que una serie de acontecimientos emergieran, murieran o siguieran instalados, como por ejemplo, la incursión de modelos educativos que dieron pie al reconocimiento de poblaciones que permanecían ocultas para la escuela pública.

Sin embargo, desde los años 50 se enunciaban en los textos escolares subjetividades como el triétnico ${ }^{3}$, las cuales irían a determinar posteriormente la manera en que la escuela abordaría el concepto de diferencia y el modo en que se objetivaría el reconocimiento por los demás en diversos grupos sociales y, sobre todo, culturales.

Así, la diversidad cultural en la escuela como la encontramos hoy, procede de cruces y mezclas de enunciados de otras épocas, entre los que destacamos el movimiento indígena y afrocolom- biano de los años 60, que se dio fundamentalmente en busca de: la recuperación de tierras ancestrales; el despliegue de un marco de derechos humanos y derechos culturales y colectivos que dio piso a la diversidad; el reconocimiento del niño como sujeto de derechos (que debe ser incluido en la escuela pero reconociéndole cada una de sus particularidades) y, finalmente, se mezcló con algunos modelos educativos que buscaban atender a la población más alejada, intentando cumplir el mandato internacional de una "educación para todos": la alfabetización y la educación rural.

\section{Educación para mantener el territorio}

Pese a que los movimientos de reivindicaciones étnicas para pueblos indígenas tienen sus antecedentes a principios del siglo $\mathrm{XX}$, su mayor fuerza comienza a darse en la década de los 60 , cuando dichos grupos, inspirados por la defensa de los territorios y el no pago de terraje, poco a poco van incluyendo nuevos intereses y encuentran en la educación la instancia predilecta, no solo para mantener su cultura, sino para conservar el territorio mismo, principal fuente de su identidad.

Es así como en 1971 surge el Consejo Regional Indígena del Cauca (CRIC), institucionalizando las organizaciones indígenas para poder dialogar con el Estado particularmente en torno a la principal problemática que padecían: la tenencia de sus tierras ancestrales. Con esto los pueblos indígenas emprendieron ese largo camino que pretendía, además de recuperar las tierras tradicionalmente ocupadas por ellos, reivindicar su historia, sus conocimientos y sus saberes (Bodnar, 2005).

Sin embargo, en el magazín dominical del diario El Espectador del año 1973, se publicaría un artículo en el que se afirmaba que la forma jurídica del Resguardo no permitía hacer frente al problema de pérdida de tierras tradicionalmente ocupadas por los indígenas, frente a ello, son procesos como la educación los que permitirían enseñar y difundir lo necesario para la defensa de sus derechos (Hernández, 1973).

En otras palabras, se diría desde entonces que era particularmente la escuela la que permitiría un aprendizaje y fortalecimiento de lo propio, en especial del derecho a la tierra. Así, se comienza a luchar por la educación como medio de acercamiento a los territorios ancestrales. Esos lazos estrechos entre territorio y educación se mantienen en la actualidad.

3 Este concepto sería una de las formas en que los textos escolares de la época del sesenta vendrían a configurar una subjetividad emergente, con la que se designarían las diferencias entre individuos a partir de sus razas, así entonces: “(...) se designa el grupo racial que tiene aportes de las tres razas primarias en proporciones más o menos equilibradas, que al decir de algunos sociólogos y antropólogos sería el tipo ideal para el pleno dominio del trópico y el verdadero creador de la futura civilización tropical” (Sánchez, 1965, p. 225). 
Esto es evidente, por ejemplo, en la normatividad, cuando menciona el territorio como uno de los principios fundamentales de la etnoeducación (Ministerio de Educación Nacional, 1995). Así mismo, es posible ver cómo se mantiene esta relación en algunos proyectos educativos de pueblos indígenas. Ello se puede observar en el video del Proyecto Etnoeducativo Majirike, de la Asociación de Autoridades Tradicionales -AATI- ASATRIZY, donde el narrador, líder indígena del Vaupés, afirma que:

"(...) Los proyectos etnoeducativos aseguran un futuro para la cultura de los grupos étnicos de Colombia. Se está preservando un conocimiento y fortaleciéndolo. Y, lo que trae como consecuencia es la conservación del territorio en el que viven. Una cosa y otra son inseparables para los indígenas colombianos, quienes desde siempre han tenido una relación activa con la naturaleza. Una relación que no destruye, que parte del conocimiento para asegurar la conservación y la permanencia del grupo" (Asociación de Autoridades Tradicionales, ASATRISY, 2010).

Con lo anterior se observa cómo la lucha por el territorio y su discurso finalmente terminó funcionando como un mecanismo productor de la práctica de la diversidad cultural en la escuela, en la medida en que se abrió paso a considerar el territorio ancestral como principal base de la identidad cultural y se insistió en la necesidad de que fuera reconocido desde la escuela diversa, no solo al enseñar sobre el uso de este bien, sino al permitir a los niños aprender sobre su derecho, enfatizando que sería en últimas lo que permitiría el rescate, mantenimiento y fortalecimiento de la cultura.

\section{"Todos los seres humanos pertenecen a la misma especie y tienen el mismo origen". Los Derechos Humanos y la identidad cultural}

En 1978, en la "Declaración sobre la raza y los prejuicios raciales", la Unesco afirmaba el derecho de todos los individuos y grupos a considerarse y ser considerados diferentes, advirtiendo, sin embargo, que la diversidad de las formas de vida y el derecho a la diferencia no pueden en ningún caso servir de pretexto a los prejuicios raciales, ni legitimar prácticas discriminatorias o fundar políticas que sean fuente de racismo.

Una década más tarde, en 1989, el Convenio No. 169 sobre pueblos indígenas y tribales en países independientes, emitido por la Organización Internacional para el Trabajo (OIT), reafirmó el derecho de los indígenas a establecer y controlar sus sis- temas educativos. Se buscaba ante todo fortalecer sus idiomas, basados en la idea de derecho que tienen todas las poblaciones a los diferentes niveles y formas de educación prestados por el Estado, argumentando que: "los pueblos indígenas tienen derecho a que la dignidad y diversidad de sus culturas, tradiciones, historias y aspiraciones queden debidamente reflejadas en la educación pública y los medios de información" (Organización Internacional del Trabajo, 1989, p. 84).

En Colombia, dos años después, la Constitución Política de 1991 afirmaría que la educación es un derecho con una función social: permitir acceso a la cultura. Así mismo, se entró a considerar que los integrantes de los grupos étnicos tenían derecho a una formación que respetara y desarrollara su identidad cultural.

A partir de esto, el congreso expidió normas otorgando una serie de derechos a aquellos que poseyeran una cultura propia, compartieran una historia y tuvieran sus propias tradiciones y costumbres dentro de la nación, distinguiéndose de otros por su identidad cultural (Ministerio de Educación Nacional, 2004).

En suma, al reconocerse los derechos colectivos, a la identidad cultural, y a los sujetos y a las sociedades como diferentes en necesidades pero iguales en reconocimientos, se fue especificando paulatinamente el discurso hasta reconocer el derecho a la educación propia, una educación que tenga en cuenta las características culturales. De esta manera, el discurso sobre los derechos se convirtió en un mecanismo que refuerza la práctica de la diversidad cultural en la escuela, pues exigió a esta institución consolidar su capacidad de atender las necesidades específicas de cada sociedad, de cada cultura.

\section{Inclusión educativa: la integración del "otro" en la escuela}

Aunque hasta el momento la diversidad cultural parece proceder del poder de las colectividades, de los grupos sociales, de las etnias y comunidades, e instaurarse en la escuela para fortalecer la identidad y mantener el territorio colectivo y el conocimiento ancestral de los pueblos, una de las fuerzas que irrumpe en este enunciado y le posibilita con más claridad su ingreso a la escuela de hoy, es la inclusión educativa, mirada centrada en el niño como sujeto de derecho.

Es así como, entre las décadas de los 50 y 80, se firmaron importantes documentos a nivel internacional entre los que se destacan el 1959 por La Asamblea General de las Naciones Unidas, donde se aprobó la Declaración de los Derechos del Niño, documento que daba reconocimiento a la libertad contra 
la discriminación y consagraba el derecho de los niños a la educación; el Pacto Internacional de Derechos Civiles y Políticos y el Pacto Internacional de Derechos Económicos, Sociales y Culturales, igualmente promovían la protección de los niños y niñas contra la explotación y afirmaban el derecho a la educación.

Por su parte, el Pacto Internacional de Derechos Culturales, publicado en 1976, hablaba de "adoptar medidas especiales de protección y asistencia en favor de todos los niños y adolescentes, sin discriminación alguna por razón de filiación o cualquier otra condición" (Naciones Unidas, 1976); y el pronunciamiento de la Convención sobre los derechos del niño, liderada por la UNESCO en 1989, recogía lo enunciado por las declaraciones sobre estos derechos (Ginebra 1924, DD del Niño 1959 y $\mathrm{DDHH})$, reafirmando y enunciando que los estados que formaran parte asegurarían el respeto a los derechos de los niños.

Con esta base, la Constitución Nacional de Colombia de 1991 incluyó como derechos fundamentales de los niños: la vida, la integridad física, la salud y la seguridad social, la alimentación equilibrada, su nombre y nacionalidad, tener una familia y no ser separados de ella, el cuidado y amor, la educación y la cultura, la recreación y la libre expresión de su opinión.

En conclusión, el enunciado de la diversidad cultural en la escuela empujó hacia la inclusión educativa, al abrir puertas al discurso del reconocimiento de la diferencia, y posicionar como objetivo la idea de que se deben reconocer las particularidades de cada sujeto. Este discurso, quiebre que se da particularmente en los años 80 y se consolida con mayor fuerza en los 90 , permitió en últimas considerar que la escuela necesitaba de un cambio para poder atender de manera pertinente a todos: niños, niñas, jóvenes, e incluso adultos, poniendo siempre por encima sus necesidades específicas, un espacio donde la cultura tuviera su lugar fundamental.

\section{Un modo de ser de la educación: la alfabetización y la educación rural}

En los planes de desarrollo de los gobiernos de la década de los 70 se expresaron ciertas situaciones que afectaban al sistema educativo, como la escasez de aulas, escuelas primarias insuficientes, maestros sin preparación, ausentismo y deserción; el analfabetismo se comenzó a reconocer como uno de los inconvenientes más "agudos” en el área rural. Bajo este panorama se propusieron proyectos como el de la "extensión de la escolaridad” el cual: “(...) se convierte en centro de la difusión de las innovaciones pedagógicas, contribuyendo a la capacitación y supervisión permanente de los maestros de las escuelas” (Pastrana, 1970-1974).

Así, el Estado intentó, por un lado, ampliar los programas de escolarización, ya que hacían "viable la consecución de ciclos completos de educación básica para determinada localidad, evitando la proliferación de escuelas de $1^{\circ}$ y $2^{\circ}$ grado" (Pastrana, 1970-1974); y por otro, ejercer control sobre los maestros y sobre los diferentes conocimientos que se iban a impartir posteriormente, develando entonces una ruptura entre el proceso "pedagógico tradicional" que la escuela venía implementado en sus prácticas, y la incursión de otros modos de ser y de actuar en ella.

A la preocupación por el analfabetismo se sumó: el hecho de que los niños eran utilizados a temprana edad como mano de obra; el desinterés de los padres sobre la educación de sus hijos, y el cuestionamiento sobre las diferencias entre patrones culturales de los estudiantes y los instrumentos utilizados para educarles (López, 1974-1978). En este contextos surgió, en la década de los 70, otra fuerza que impulsaría la instalación de la diversidad en la escuela: la educación rural.

Este modelo aparecería gracias al interés de educar a las poblaciones de estas zonas, buscando también incrementar sus oportunidades de participación. De este modo, el reconocimiento de una subjetividad infantil distinta, el niño campesino, empezó a considerarse como importante en el marco de los asuntos del Estado y la sociedad.

Para 1976 el modelo de Escuela Nueva irrumpió en la educación pública como un esquema diseñado para atender, en una sola aula, a todos los niños y jóvenes de una zona determinada, garantizándoles promoción flexible y escolaridad completa. Esta aparición, junto con otras que marcaron presencia en la zona rural del país -Mapa Educativo, Centros Experimentales Piloto, Escuelas Normales, educación contratada, educación de la comisaría (Fondo Educativo Regional), Instituto Colombiano de Bienestar Familiar (ICBF), Dirección General de Capacitación y Dirección General de Administración-, se configuró como un quiebre que agrandaría la fisura del proyecto de unidad nacional dentro del proceso educativo, impulsando así el enunciado de la diversidad cultural en la escuela.

En suma, el cruce de varias tensiones, la preocupación por el analfabetismo y la falta de atención a las poblaciones rurales, donde era marcada la deserción y la falta de cobertura escolar, exigiría la implementación de otros modos de actuar en el proceso de enseñanza-aprendizaje y el aula escolar. Con proyectos 
como el de tecnología educativa, las escuelas diversificadas, el establecimiento de currículos que atendieran el futuro laboral de los estudiantes, y programas como Escuela Nueva, se fortalecería la idea de la necesidad del reconocimiento de las diferencias académicas y culturales de los estudiantes.

\section{El devenir de la diversidad cultural en la escuela: las paradojas irresolubles}

La escuela pública, una institución que hasta los años setenta se caracterizaba por reproducir relaciones jerárquicas de poder, enseñar normas de comportamiento, una forma de conocimiento y un sistema de creencias; que se definía desde un espacio cerrado, creado para adoctrinar, disciplinar y formar sujetos de una gran nación; que estaba para homogeneizar e invisibilizar lo diverso bajo su intención de uniformar, estandarizar y parametrizar, es atravesada por varias fuerzas y discursos que buscaban incluir a la diversidad cultural en ella.

Este cambio permite hacer múltiples preguntas: ¿Cómo cambia la escuela al verse frente al enunciado de la diversidad cultural?; ¿qué tanto debe la diversidad cultural a la escuela?; ¿qué tanto debe la escuela de hoy a la diversidad cultural? ¿En este proceso, la escuela juega un papel central porque en el núcleo del discurso se encontró un problema pedagógico?

Sin ánimo de agotar el tema respondiendo todas las preguntas que han quedado sin resolver, éstas, junto con los resultados de la investigación, expuestos en los apartados anteriores, permiten plantear unas conclusiones seguidas de tres paradojas que caracterizan a la escuela de hoy, y que a la vez definen la forma en que la diversidad cultural se relaciona con esta institución.

Por un lado, vemos que este cambio en el modo de ser de la escuela ha producido, o permitido el fortalecimiento de nuevas subjetividades -aunque sea a nivel discursivo o teórico-, por ejemplo, el niño diverso, el maestro para la diversidad, el niño diferente, el niño dimensional (desde el sicoanálisis), el niño social, entre otros, logrando particularmente un reconocimiento del "otro", un posicionamiento del "otro", del sujeto históricamente invisibilizado y negado.

Esto ha sido posible particularmente gracias a que la escuela ha generado nuevos saberes basados en las políticas educativas (proyectos etnoeducativos, cátedra de estudios afrocolombianos), discursos que permean las áreas reconociendo el valor de lo diferente, lo cual, sin embargo, permite afirmar que la relación entre diversidad cultural, saberes escolares, políticas del conocimiento en educación y pedagogía, está basada en múltiples lazos que se dirigen en diversos sentidos; la escuela ha fortalecido el discurso de la diversidad, así como la diversidad ha enriquecido a la escuela.

Sin embargo, nos preguntamos qué tanto se ha naturalizado esta idea de la diversidad cultural en la escuela, cuando observando proyectos educativos institucionales que en su mayoría no tienen en cuenta las particularidades y siguen promoviendo las mismas prácticas tradicionales: enseñando áreas fundamentales establecidas por ley (matemáticas, castellano, ciencias naturales y ciencias sociales) y definidas por un grupo étnico occidental. También nos cuestionamos qué tanto se ha trabajado esta idea, cuando el conocimiento que se imparte en la escuela está basado en las ciencias naturales, en un ideal positivista, en una clasificación de las cosas que es propia de occidente, no de las culturas diversas.

Con esto no se quiere decir que las prácticas educativas actuales no sean lo que deberían, pues estamos seguros de que no existe tal deber ser, de que no existe una única manera, una verdad. Con esto buscamos generar nuevas cuestiones, seguir problematizando la diversidad cultural y la escuela de hoy.

Si bien la diversidad se ha convertido en un saber escolar y ha generado nuevos saberes que han incidido en el fortalecimiento de subjetividades y de conocimientos en educación y pedagogía, este acontecimiento sigue marcado por tensiones (identidad multivariada, cultura y emergencia de otras, contexto, entre otras) y fisuras que también lo hacen su modo de ser en la época actual. Por eso es necesario destacar tres paradojas irresolubles que permiten finalizar el presente escrito, dejándolo abierto a nuevas incursiones investigativas.

La primera de ellas muestra que a la escuela, una institución diseñada para disciplinar, normalizar y homogeneizar, con un solo PEI y un solo currículo, se le asignan tareas y obligaciones de asistencia y "educación para todos", para las diferentes etnias y grupos poblacionales dispersos, minoritarios; debe atender la diversidad y cumplir con objetivos que van más allá de sus límites, la función social de la inclusión de las diversidades.

Sin embargo, pese a los intentos por incluir lo diverso en la escuela, se advierte que ésta sigue marcada por un efecto homogeneizador, cuando lo diverso, culturalmente hablando, es atajado por unas necesidades primarias, definidas desde una cultura como derechos fundamentales, que cobijan a toda la población 


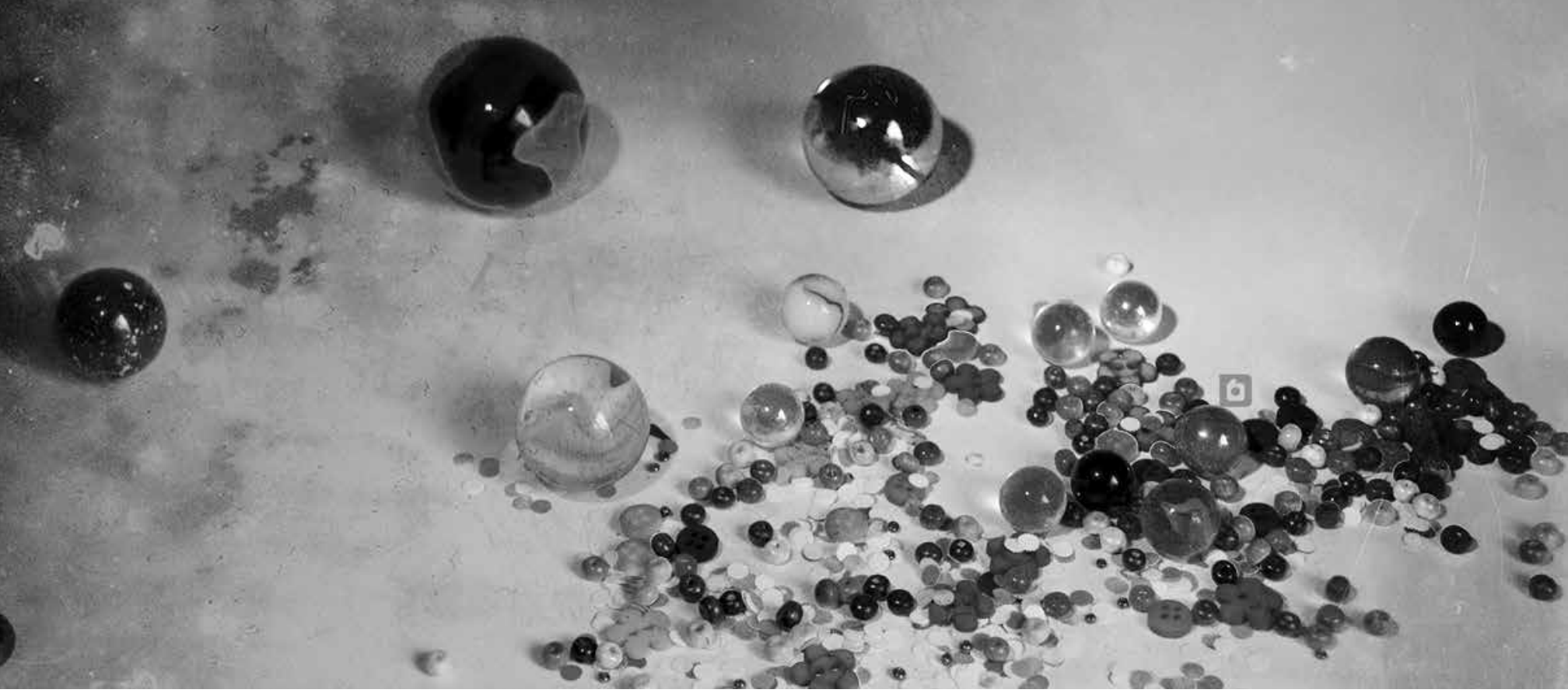

mundial, lo cual nos guía hacia una segunda paradoja: la tensión existente e irresoluble entre la intención de fortalecer una identidad cultural colectiva y los derechos humanos individuales.

Finalmente la tercera y última paradoja, está basada en el interés de los grupos étnicos por ser considerados como ciudadanos de derechos, y su lugar dentro de la obligación internacional de inscribir a sus niños en las escuelas definidas tradicionalmente desde una cultura externa.

\section{Conclusiones}

Con este recorrido arqueológico-genealógico sobre el archivo histórico de Colombia, se puso en evidencia la manera en que el concepto de diversidad cultural emergió en la institución educativa y sus giros durante el tiempo. Mientras en los 60 la escuela se acercaba de formas muy diferentes a la diversidad, pues concebía las diferencias regionales y raciales únicamente como parte de su proyecto unificador nacional, en los 70 la institución empezó a ser receptora y productora de nuevos sujetos y nuevas prácticas, en la medida en que aparecían diversos enunciados a nivel internacional, como el reconocimiento de los Derechos Humanos, la no discriminación racial o el ideal objetivado de incluir a todos los niñas y niñas en la escuela.

A partir de la década de los 70, prácticas provenientes de diversos campos fueron las principales encargadas de ir forjando el enunciado de la diversidad cultural como se conoce hoy, tales como: el movimiento reivindicativo por el derecho a las tierras ancestrales de los grupos étnicos; el enunciado, tejido desde mediados del siglo XX, alrededor de los Derechos Humanos y los derechos colectivos; el reconocimiento internacional a la necesidad de atender las necesidades y características individuales de cada sujeto; y, finalmente, algunos modelos educativos que comenzaron a tratar de llegar a las poblaciones más apartadas bajo el lema de una "educación para todos".

Desde estos primeros años -inicios de los 70-, la diversidad cultural apareció fundamentalmente bajo el nombre de etnoeducación, y con ella toda una serie de normas, discursos y prácticas la han venido definiendo hasta la actualidad. Pasados 20 años, el discurso etnoeducativo comenzó a mostrar quiebres y, a pesar de que el enunciado seguía siendo el mismo, y funcionaba bajo los mismos principios fundamentales, apareció un nuevo elemento que tomaría fuerza en la diversidad: la interculturalidad.

Sin embargo, a pesar de todas estas fuerzas que configuraron con firmeza el enunciado de la diversidad, este discurso, como cualquier acontecimiento social, no deja de tener tensiones, algunas de ellas irresolubles, que solo con el tiempo se podrán desaparecer, mutar o robustecerse para dar vida al modo de ser de la escuela del futuro.

Así, la diversidad cultural como acontecimiento, como un artilugio que intentó el reconocimiento de la diferencia y de la diversidad étnica y cultural, entre otras, y que empezó a irrumpir en la escuela pública colombiana a mediados de los 70, transformando la escuela, el maestro, el niño, el currículo, entre otros, poco a poco se fue convirtiendo en un saber escolar, esto es, en unas prácticas de enseñanza que incidieron en la constitución de nuevas subjetividades, en la configuración de unos objetos de conocimiento distintos, otras formas de evaluación y de formulación del currículo. 


\section{Referencias}

Alcaldía Mayor de Bogotá. (2005). Políticas culturales distritales 2004-2006. Bogotá: Panamericana.

Alfonso, L. A. (1982). El proyecto de educación para territorios Nacionales. Revista Educación Territorios Nacionales, 1-7.

Amaya, T. S. (2009). Aproximación a un estudio genealógico de la evaluación en Colombia, siglo XX. Revista latinoamericana. Ciencias Sociales, Niñezy Juventud, 1 - 37.

Asamblea General de las Naciones Unidas. (1959). Convención sobre los Derechos del Niño. Décimo cuarto período de sesiones.

Asociación de Autoridades Tradicionales ASATRISY. (2010). Proyecto Etnoeducativo Majirike de la AATI AS ATRIZY. Obtenido en 2012, desde http://www.youtube.com/watch?v=sSeKy2N_-gA

Boom, A. M. (1994). Currículo y Modernización. Bogotá: Tercer Milenio.

Bodnar, Y. (2005a). Diversidad cultural versus prácticas estadísticas asimilacionistas. Seminario Internacional: Pueblos Indígenas de Latinoamérica y del Caribe: Sistemas de Información y Gestión Pública, organizado por La Comisión Nacional para el Desarrollo de los Pueblos Indígenas -CDI- y PNUD. México.

Congreso de la República. (2006). Ley 1098. Diario Oficial. No. 46.446, miércoles 8 de noviembre de 2006. Bogotá.

Escuela de Educación Diferencial. (2009). Revista Latinoamericana de Educación Inclusiva, 3 (2).
Ferro, M. C. (1982). Análisis de una experiencia: La Misión Alemana. Red Académica, 1 - 45.

Grueso Bonilla, A. (2010). La interculturalidad más allá de lo étnico. Revista Internacional Magisterio. Educación y Pedagogía (46), 20-21.

Hernández, F. (1973). Integración de Motilones. El Espectador. Magazín Dominical, 7 de Noviembre.

Ministerio de Cultura. (1997). Ley 397. Bogotá, Colombia.

Ministerio de Educación Nacional. (1963). Decreto 1710. Por el cual se adopta el plan de estudios de la educación primaria colombiana. Diario Oficial. No. 31169. Bogotá.

Ministerio de Educación Nacional. (1976). Decreto número 088. Diario Oficial. No. 34495, 22 de Enero. Bogotá, Colombia.

Ministerio de Educación Nacional. (1978). Decreto 1142. Obtenido desde http://www.mineducacion.gov.co/1621/articles-102752_archivo_pdf.pdf

Ministerio de Educación Nacional. (1995). Decreto 804. Diario Oficial. No. 41.853, 18 de Mayo. Bogotá, Colombia.

Ministerio de Educación Nacional. (2004). Directiva Ministerial. No. 11, 19 de Mayo. Bogotá, Colombia.

Ministerio de Educación Nacional. (2005). Lineamientos de política para la atención educación a la población vulnerable. Bogotá. 
Naciones Unidas. (1989). Convención sobre los derechos del niño.

Naciones Unidas. (Marzo de 1990). Conferencia mundial sobre educación para todos. Declaración Jomtiem. Jomtiem.

Organización Internacional del Trabajo. (1989). Convenio No. 169. Sobre pueblos indígenas y tribales en países independientes. Lima: OIT.

Pardo, S. (1976). ¿Las escuelas son obsoletas? El Tiempo. 29 de Enero. p. $7 \mathrm{D}$.

Pastrana, M. (1970-1974). Plan Nacional de Desarrollo. Bogotá.

Presidencia de la República. (1991). Constitución Politica de Colombia. Recuperado en marzo de 2012, desde: http:/ /wsp.presidencia.gov.co/ Normativa/Documents/Constitucion-Politica-Colombia.pdf

Orbitastarmedia. (2000). Pronunciamiento latinoamericano. Obtenido en 2012, desde: http://orbita.starmedia.com/ constructivismo/pronunciamiento.htm

Ramón, H. J. (1951). Geografía Superior de Colombia. 5a. Edición. Bogotá: Librería Stella, Colección La Salle.

Rodríguez Echeverry, N. (2006). Estado del arte de la investigación sobre las comunidades de afrodescendientes y raizales en Bogotá D.C. Bogotá: Instituto Distrital de Cultura y Turismo.
Sánchez Eusse, H. (1965). Elementos de la Geografía general y de Colombia. Bogotá: Bedout.

Sánchez-Araya, R. (1969). Misión de asistencia Técnica. Bogotá: Unesco.

Corte Suprema de Justicia (1998). Sentencia 510, SU-510/98.

Turbay, J. C. (1978-1982). Plan nacional de desarrollo. Bogotá.

UNESCO. (1978). Declaración sobre la raza y los prejuicios raciales. Obtenido desde: http://portal.unesco.org/es/ev.php-URL_ ID=13161\&URL_DO=DO_TOPIC\&URL_SECTION=201.html

UNESCO. (2000). Declaración de Dakar. Obtenido en 2012, desde: http://www.unesco.org/education/efa/ed_for_all/dakfram_spa. shtml

UNESCO. (2002). Declaración universal sobre la diversidad cultural. Johannesburgo.

UNESCO. (2005). Politicas educativas de atención a la diversidad. Brasil, Chile, Colombia, México y Perú. Santiago, Chile: OREALC.

UNESCO. (2008). Diversidad cultural e interculturalidad en educación superior. Experiencias en América Latina. Caracas.

UNESCO. (2008). Educación y diversidad cultural. Lecciones desde la práctica innovadora en América Latina. Santiago de Chile: Pehuén Editores. 


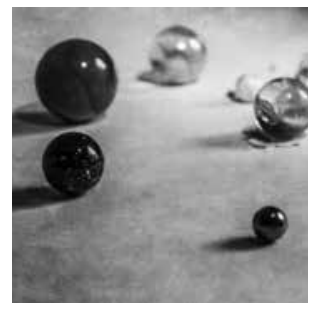

